\title{
The VLBI Core in Radio Loud AGNs
}

\author{
D. R. Jiang, Xinwu Cao and J. F. Zhou
}

Shanghai Astronomical Observatory, Chinese Academy of Sciences, Shanghai, National Astronomical Observatories, China

\begin{abstract}
.
We present statistical results on VLBI cores from large VLBI samples at $\mathrm{C}, \mathrm{L}$ and $\mathrm{S} / \mathrm{X}$ bands. Most of the results are consistent with the inhomogeneous jet model. The magnetic field distribution and particle density in the inhomogeneous jet model were estimated. We also found that the flux densities and luminosities of the cores at $5 \mathrm{GHz}$ were correlated with those at optical band in the rest-frame.
\end{abstract}

\section{Introduction}

In active galactic nuclei (AGNs), the core-jet morphology is common on VLBI scales. To describe the main structure in a compact jet, model fitting to the visibility function is used to estimate the angular sizes, flux densities and relative positions of the components. The VLBI cores are often unresolved in the ground VLBI observations, and usually have flat or inverted spectra over the radio band. It is believed that the VLBI core is the base of the compact jet in an AGN. Here, we present some statistical results on the large VLBI samples.

\section{Data}

The fitted data at $\mathrm{C}$ and $\mathrm{L}$ band come from the Pearson-Readhead+CaltechJodrell sample. The corresponding $\mathrm{S} / \mathrm{X}$ band data are from the simultaneous dual-frequency VLBA observations of radio reference frame sources.

The fitted parameters include the flux density, major axis $a$ and axis ratio $b$ $\left(b=a x i s_{\text {minor }} /\right.$ axis $\left._{\text {major }}\right)$ of the components. We identified a core according to the structure and the spectral index of two images at different frequencies. The most compact component was believed as the core when there were images only at one frequency. Definitions $s_{\nu} \propto \nu^{-\alpha}, \theta_{d}=a \sqrt{b}$, and $\theta_{d}($ core $) \propto \nu^{-p}$ are used in this paper, where $\theta_{d}$ is the mean angular size of the core, $p$ is the frequency index of a core angular size.

\section{Results and discussion}

1) There are 62 sources with non-zero $a$ and $b$ at $\mathrm{C} / \mathrm{L}$ bands in the PR+CJ1 sample, and 102 sources in the radio reference frame sources at $\mathrm{S} / \mathrm{X}$ bands. The frequency ranges of the above two samples overlap, we combined the data of the 
two samples for the statistics. Here the spectral index $\alpha$ (core) is derived directly from the model flux densities at the two frequencies without beam correction. The frequency index $p$ is estimated from the mean core sizes at two frequencies. The median values of $\alpha$ (core $)$ and $\mathrm{p}$ for this combined sample are 0.11 and 1.06 . The median values of the core sizes at $1.66,2.32,5.0$ and $8.55 \mathrm{GHz}$ can be fitted by $\theta_{d} \propto \nu_{o b}^{-0.93}$.

The statistical results of the angular size and the spectral index of VLBI core are consistent with the inhomogeneous jet model. We can use these results to estimate the distributions of the magnetic field and the number density of relativistic electrons assuming $B(r) \propto r^{-m}$ and $n_{e}(r) \propto r^{-n}$ (Königl 1981). The median values of $m$ and $n$ are $0.87,1.95$ respectively.

2) Gurvits et al (1999) estimated the angular size of the compact jet $\left(\theta_{j e t}\right)$ from the VLBI images at $5 \mathrm{GHz}$. They defined the angular size of a source as the distance between the core and the most distant component which has a peak brightness greater than or equal to $2 \%$ of that of the core. There are 210 different sources, which have measurements of both the major axis angular size of the core and the compact jet angular size. We found that these two angular sizes are correlated at the confidence level $95 \%$, and the mean ratio of the major angular size of the core to the angular size of the compact jet is 0.18 . The inhomogeneous jet model (Cawthorne 1992) with above $m$ and $n$ value predicted that this ratio would be about 0.18 , which is in agreement with the observed ratio.

3) The relationship between the radio emission of the VLBI core and the optical emission is explored in this work. A spectral index 0.7 in the optical band is assumed for the $\mathrm{K}$ correction and also for the estimation of flux density at $\mathrm{v}$ band of CJ2 sample. The fluxes of the VLBI core at $5 \mathrm{GHz}$ are interpolated for the sources observed at $\mathrm{S} / \mathrm{X}$ bands, $\alpha($ core $)=0.0$ was assumed for the $\mathrm{K}$ correction when the fitted data at only one frequency was available. We found that the radio emissions of the VLBI core are strongly correlated with the optical emissions in the rest frame for quasars and BL Lacs. the Spearman rank correlation between two fluxes is at the level $99.99 \%$. There is no correlation between these two emissions for the radio galaxies at low redshift.

VSOP observations have the higher resolution than those at ground VLBI array; a statistical investigation of a large sample will reduce the effects of the dependence of the flux density of the VLBI core on the resolution.

\section{References}

Cawthorne, T. V., 1992, in Beams and Jets in Astrophysics edited by P. A. Hughes (Cambridge University Press), 205

Gurvits, L. I., Kellerman, K. I., Frey, S., 1999, A\&A, 342, 378

Königl, A., 1981, ApJ, 243, 700 